\title{
A Silicon Implementation of the Fly's Optomotor Control System
}

\author{
Reid R. Harrison \\ Christof Koch \\ Computation and Neural Systems Program 139-74, California Institute of Technology, \\ Pasadena, CA 91125, U.S.A.
}

Flies are capable of stabilizing their body during free flight by using visual motion information to estimate self-rotation. We have built a hardware model of this optomotor control system in a standard CMOS VLSI process. The result is a small, low-power chip that receives input directly from the real world through on-board photoreceptors and generates motor commands in real time. The chip was tested under closed-loop conditions typically used for insect studies. The silicon system exhibited stable control sufficiently analogous to the biological system to allow for quantitative comparisons.

\section{Introduction}

Flies use visual motion information to estimate self-rotation and generate a compensatory torque response to maintain stability during flight. This wellstudied behavior is known as the optomotor response. It is interesting from an engineering standpoint because it extracts relevant information from a dynamic, unstructured environment on the basis of passive sensors and uses this information to generate appropriate motor commands during flight. This system is implemented in biological hardware that is many orders of magnitude smaller and more power efficient than charge-coupled device (CCD) imagers coupled to a conventional digital microprocessor.

Much of the computation underlying the optomotor control system is performed by the horizontal system (HS) cells of the fly visual system (Geiger \& Nässel, 1981, 1982; Egelhaaf, Hausen, Reichardt, \& Wehrhahn, 1988; Hausen \& Wehrhahn, 1990; Egelhaaf \& Borst, 1993). The three HS cells (HSN, HSE, and HSS) belong to a group of 50 to 60 giant tangential neurons having large dendritic arborizations in the lobular plate region of the optic lobe (Hausen, 1981, 1982, 1984; Hengstenberg, 1982; Krapp \& Hengstenberg, 1996). HS cells are nonspiking neurons that are depolarized by full-field visual motion from the front to the back of the eye and hyperpolarized by back-to-front motion. Each HS cell integrates signals from an ipsilateral retinotopic array of elementary motion detectors (EMDs), units in the medulla that estimate local motion in small areas of the visual field. 
The HS cells synapse onto descending, spiking neurons, which relay information to the motor centers of the thoracic ganglion.

We built a single-chip integrated imager and analog computer that mimics the fly optomotor control system and produces compensatory motor signals in real time. Based on earlier work by Mead and colleagues, we use standard CMOS field-effect transistors operating in the subthreshold regime where the source-drain current through the transistor is exponentially related to its gate voltage (Mead, 1989). Similar to neural systems, all our computations are performed in parallel by analog and distributed circuits operating in continuous time. There is no software; the algorithm is entirely specified by the circuit architecture.

Several researchers have built hardware models of insect visual systems with analog VLSI (Andreou \& Strohbehn, 1990; Delbrück, 1993; Sarpeshkar, Bair, \& Koch, 1993; Etienne-Cummings \& Van der Spiegel, 1996; Moini et al. 1997), discrete analog hardware (Franceschini, Pichon, \& Blanes, 1992), and traditional CPUs with CCD cameras (Srinivasan, Chahl, \& Zhang, 1997; Lewis, 1998), many of which have been incorporated into mobile robots. The comparisons made to biology have been qualitative in nature. We present here a more rigorous approach to neuromorphic engineering in which hardware models are evaluated by directly replicating experiments originally performed on their biological counterparts.

\section{Description of Hardware Model}

Our motion detector architecture is a delay-and-correlate scheme similar to that that first proposed by Hassenstein and Reichardt (1956) to explain beetle behavior, where a temporally bandpassed signal from one photoreceptor is multiplied with the delayed bandpassed signal from an adjacent photoreceptor. The result is subtracted from the mirror-symmetric operation to remove the directional insensitive component. The output of many such EMDs sensitive to motion at different locations is added and low-passfiltered to give the final output signal that the insect uses to stabilize its flight. There is good evidence that correlation-based EMDs form the basis of the optomotor system in the fly (Reichardt \& Egelhaaf, 1988; Borst \& Egelhaaf, 1989).

Our chip consists of an array of photoreceptors and EMDs whose outputs are summed (see Figure 1). A lens mounted over the chip focuses the image of the outside world onto the silicon surface. Each elementary motion detector uses photodiodes as light sensors. We use a four-transistor adaptive photoreceptor circuit developed by Delbrück (Delbrück \& Mead, 1996) that produces a continuous-time output voltage proportional to the logarithm of light intensity (see Figure 2a). This circuit has a temporal low-pass characteristic with a cutoff frequency that can be set with a bias voltage. The photoreceptor is connected to a temporal derivative circuit (Mead, 1989) (see Figure 2b), which has a high-pass behavior. Transient firing, charac- 


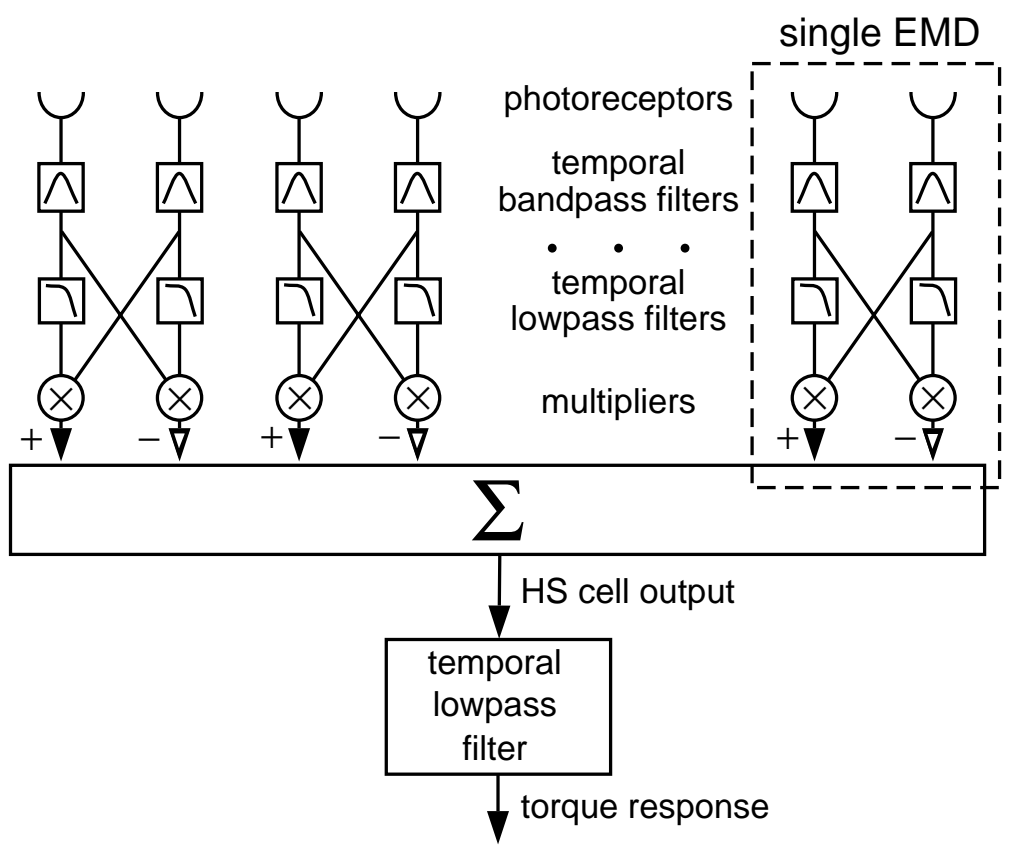

Figure 1: Model of the fly optomotor system implemented in silicon. Photoreceptor outputs are temporally bandpass filtered and fed into HassensteinReichardt elementary motion detectors (EMDs) consisting of first-order lowpass filters ( $\tau=40 \mathrm{~ms}$ ) followed by multipliers. The HS cell is modeled simply as a spatial summation of opponent EMDs. The HS cell response is passed through an off-chip low-pass filter $(\tau=680 \mathrm{~ms})$, mimicking the behavior of the thoracic motor centers, to generate the torque response. The silicon implementation includes 13 EMDs with integrated photoreceptors on a single $2.2 \mathrm{~mm} \times$ $2.2 \mathrm{~mm}$ chip fabricated in a standard $2.0 \mu \mathrm{m}$ CMOS process.

teristic of a temporal high-pass response, has been observed in fly laminar cells that receive input from retinal photoreceptors (Weckström, Juusola, \& Laughlin, 1992). Together, the low-pass filtering of the photoreceptor and the high-pass filtering of the temporal derivative circuit form a bandpass filter, which improves performance by eliminating DC illumination (which contains no motion information) and attenuating high-frequency noise such as the $120 \mathrm{~Hz}$ flicker of AC incandescent lighting. These bandpass filters were set to attenuate frequencies below $2.8 \mathrm{~Hz}$ and above $10 \mathrm{~Hz}$.

The temporal derivative circuit relies on a high-gain differential amplifier in a negative feedback configuration to keep the voltage on the capacitor equal to the input voltage. As the capacitor charges and discharges to main- 
a Photoreceptor

\section{b Temporal Derivative Circuit}

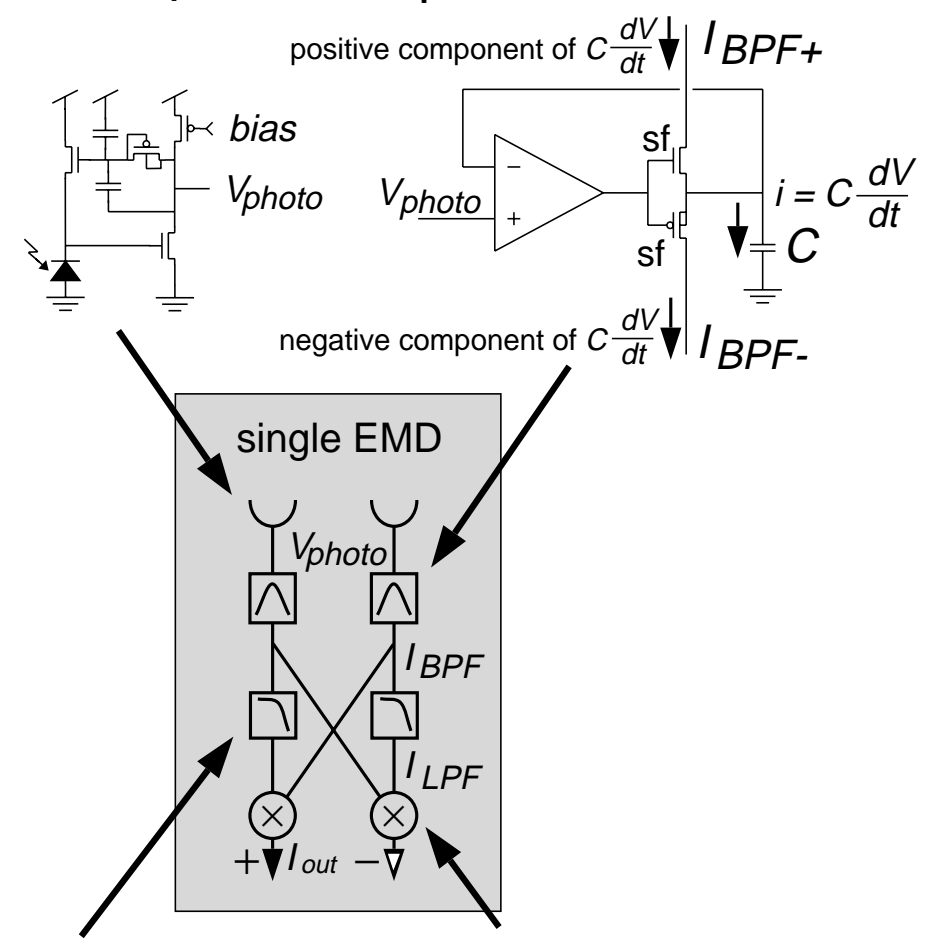

c Low-Pass Filter

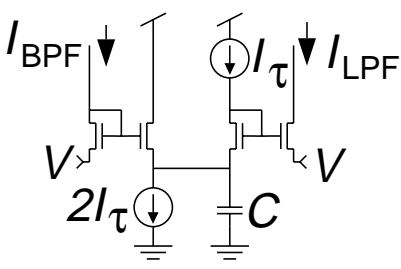

$$
\begin{gathered}
\tau \frac{d I_{L P F}}{d t}+I_{L P F}=I_{B P F} \\
\tau=\frac{C U_{T}}{I_{\tau}}
\end{gathered}
$$

d Multiplier

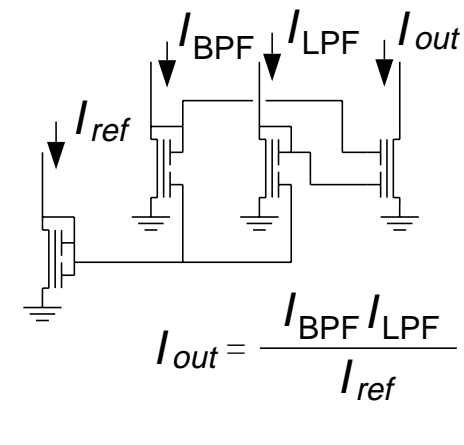

tain this equality, the currents through the two source follower transistors (labeled "sf" in Figure 2b) may be measured. The outputs of the temporal derivative circuit are these two unidirectional currents, which are proportional to the positive and negative components of temporal derivative of the input voltage. This resembles the ON and OFF channels found in many 
biological visual systems. One study suggests ON and OFF channels are present in the fly (Franceschini, Riehle, \& Nestour, 1989), but the evidence is mixed (Egelhaaf \& Borst, 1992).

We use the phase lag inherent in a first-order low-pass filter as a time delay. The currents from the temporal derivative circuit are passed to currentmode first-order low-pass filter circuits (see Figure 2c) (Himmelbauer, Furth, Pouliquen, \& Andreou, 1996). These are log-domain filters that take advantage of the exponential behavior of field-effect transistors (FETs) in the subthreshold region of operation. Note that two filters are needed for each EMD: one for the ON channel and one for the OFF channel, which are processed in parallel. The time constant of the filters is controlled with a bias current that can be set externally. This time constant can be changed to tune the EMD to a specific optimal temporal frequency. For all the experiments described in this article, we fixed this time constant to $40 \mathrm{~ms}$. This gave our chip a maximum temporal frequency sensitivity of $4 \mathrm{~Hz}$, similar to motion-sensitive neurons in flies (O'Carroll, Bidwell, Laughlin, \& Warrant, 1996).

To correlate the delayed and nondelayed signals for motion computation, we use a current-mode multiplier circuit (see Figure $2 \mathrm{~d}$ ). This circuit takes advantage of the exponential behavior of subthreshold FETs to perform a computation. Two diode-connected FETs convert the input currents into log-encoded voltages. The weighted sum of these voltages is computed with the capacitive divider on the floating gate of the output transistor, and this transistor exponentiates the summed voltages into the output current, completing the multiplication. Any trapped charge remaining on the floating gates from fabrication is eliminated by exposing the chip to ultraviolet light, which imparts sufficient energy to the trapped electrons to allow passage through the surrounding insulator. This circuit represents one of a family of floating-gate MOS translinear circuits developed by Minch that are capable of computing arbitrary power laws with current-mode signals (Minch, Diorio, Haster, \& Mead, 1996). After multiplication is performed in both the ON and OFF channels, these two signals are summed.

Figure 2: Facing page. EMD subcircuits. (a) Photoreceptor. This circuit produces an output voltage proportional to the logarithm of light intensity. (b) Temporal derivative circuit. In combination with the low-pass filter inherent to the photoreceptor, this forms a temporal bandpass filter with a current-mode output. (c) Low-pass filter. The time constant of this first-order filter is determined by the bias current $I_{\tau}$ (which is set by a voltage supplied from off-chip) and the capacitance $C$. (d) Multiplier. The devices shown are floating-gate nFET transistors with capacitive inputs. The two inputs couple to the floating gate, forming a capacitive divider. The input transistors are diode connected, which converts the input currents into log-encoded voltages. The capacitive divider on the output transistor computes a weighted sum of these voltages. The output transistor produces a current proportional to the exponental of this sum. 
One entire EMD (left and right channels) consists of 31 transistors and 25 capacitors with $8.0 \mathrm{pF}$ of total capacitance. Each EMD takes $0.044 \mathrm{~mm}^{2}$ of silicon area in a $2.0 \mu \mathrm{m}$ CMOS process, including the integrated photoreceptors. By operating most of the transistors in the subthreshold regime, we achieve extremely low power dissipation (approximately $7.5 \mu \mathrm{W}$ per elementary motion detector).

We built a simple model of the HS cell by constructing a one-dimensional array of 13 complete EMDs and linearly summing their outputs. This is easily achieved due to the current-mode nature of the EMD output signals; we simply tie all the wires together. Dendritic integration is likely to be nonlinear. When stimulus size is increased, motion-sensitive neurons in the fly exhibit gain control, where the response increases less than linearly with size (Borst, Egelhaaf, \& Haag, 1995; Single, Haag, \& Borst, 1997). Our model does not include this nonlinear size dependence, but all our experiments use patterns of fixed size.

Figure 3 shows the response of the EMD array to sinusoidal gratings with varying temporal frequencies. Data from a fly wide-field motion neuron is shown for comparison. Both motion detectors show temporal frequency tuning that is characteristic of the delay-and-correlate motion detector (Adelson \& Bergen, 1985). The greater width of the fly tuning curve is probably due to adaptation of the EMD low-pass filter time constant (de Ruyter van Steveninck, Zaagman, \& Mastebroek, 1986; Borst \& Egelhaaf, 1987; Clifford, Ibbotson, \& Langley, 1997). Also, bandpass filtering of the photoreceptor signals attenuates low- and high-frequency stimuli in our VLSI model. We have measured the response of our EMD circuit to drifting sinusoids while varying spatial frequency and direction, and have shown that the circuit exhibits tuning similar to that observed in insect motion-sensitive neurons (data not shown; see Harrison \& Koch, 1998).

\section{Measuring the Optomotor Response}

3.1 Experiments Previously Performed on Flies. Warzecha \& Egelhaaf (1996) recently characterized the optomotor behavior of the fly under closedloop conditions. A female sheepfly (Lucilia cuprina, Calliphoridae) was rigidly attached to a meter that measured yaw torque produced while the fly attempted to turn in response to visual stimuli (see Figure 4a), reducing the fly's behavior to a single degree of freedom. Vertical bars were presented to a large region of the fly's visual field and could be drifted clockwise or counterclockwise. In closed-loop experiments, the fly's yaw torque was measured in real time and scaled by a constant gain term to yield angular velocity. This simulates the observed dominance of air friction in determining the instantaneous angular velocity in flies (Reichardt \& Poggio, 1976). The fly's simulated angular velocity was subtracted from the angular velocity imposed by the experimenter. The resulting signal was used to control the drift rate of the visual stimulus. This simulated free-flight conditions and allowed evaluation of the optomotor system performance.

The imposed motion schedule consisted of $3.75 \mathrm{~s}$ of zero imposed motion, then $7.5 \mathrm{~s}$ of clockwise rotation at 44 degree $\mathrm{s}^{-1}$. Figure $5 \mathrm{a}$ shows the torque 


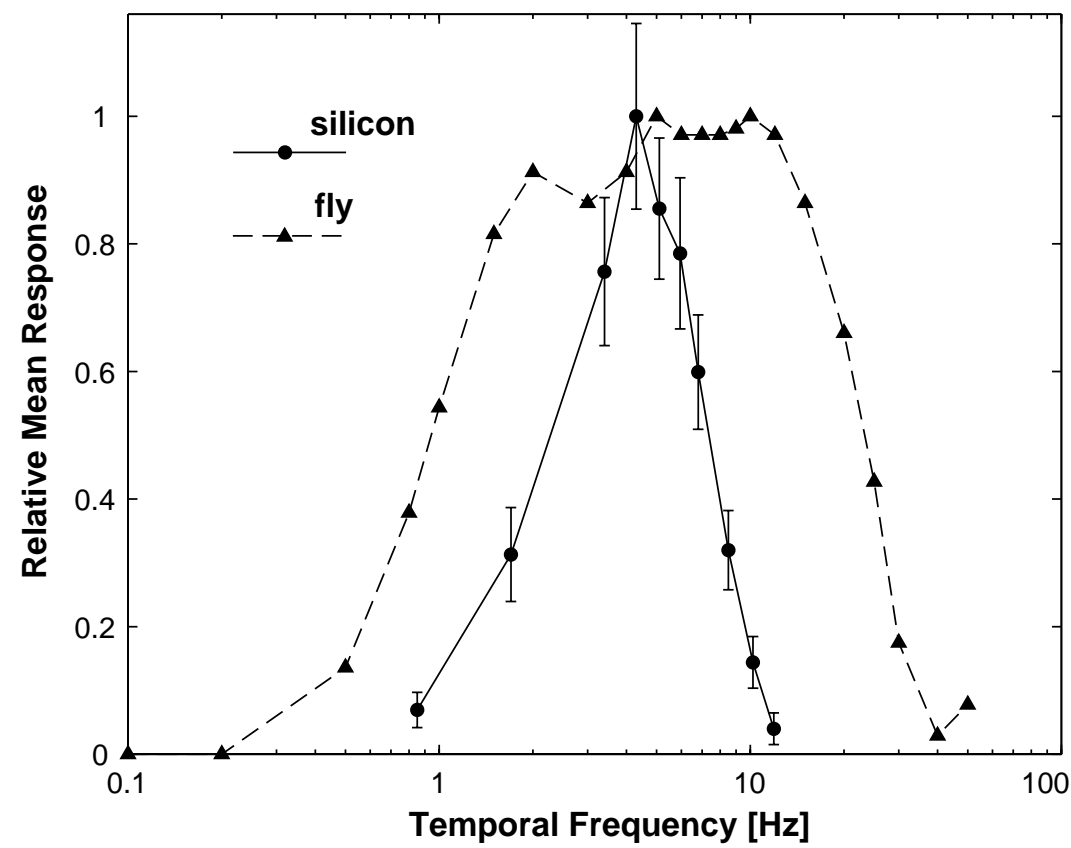

Figure 3: Normalized temporal frequency response of silicon (circles) and fly (triangles) motion detectors. Both systems exhibit temporal frequency tuning characteristic of correlation-based motion detection schemes. (Temporal frequency is proportional to velocity for constant spatial frequency.) The width of the fly tuning curve is probably due to adaptation of the EMD low-pass filter time constant (Clifford et al., 1997). The stimulus used for the silicon EMD array was a sinusoidal grating with a spatial frequency of 0.03 cycles $\mathrm{deg}^{-1}$, resulting in two- to three-pattern wavelengths across the photodetector array. Error bars denote standard deviation of chip response during an $800 \mathrm{~ms}$ recording interval ( $2 \mathrm{kHz}$ sampling rate), indicating some residual pattern dependence. Fly data are normalized mean spike rate (spontaneous activity subtracted) taken from an unspecified lobular plate wide-field motion neuron in the blowfly (Calliphora erythrocephala). Fly data redrawn from O'Carroll et al. (1996).

data and resulting stimulus position for an individual trial. Figure $5 \mathrm{~b}$ shows the averaged data over 139 trials in a total of five animals. (See Warzecha \& Egelhaaf, 1996, for details on the experimental protocol.)

The fly is able to stabilize its flight and cancel out most of the imposed motion. Simulation results suggest that the nonmonotonic temporal frequency response of Reichardt motion detectors results in greater stability for the optomotor control system (Warzecha \& Egelhaaf, 1996). The individual trials show an oscillatory component to the torque response around $2 \mathrm{~Hz}$. This oscillation is not linked to the stimulus since it is not present in the average torque trace. Oscilla- 

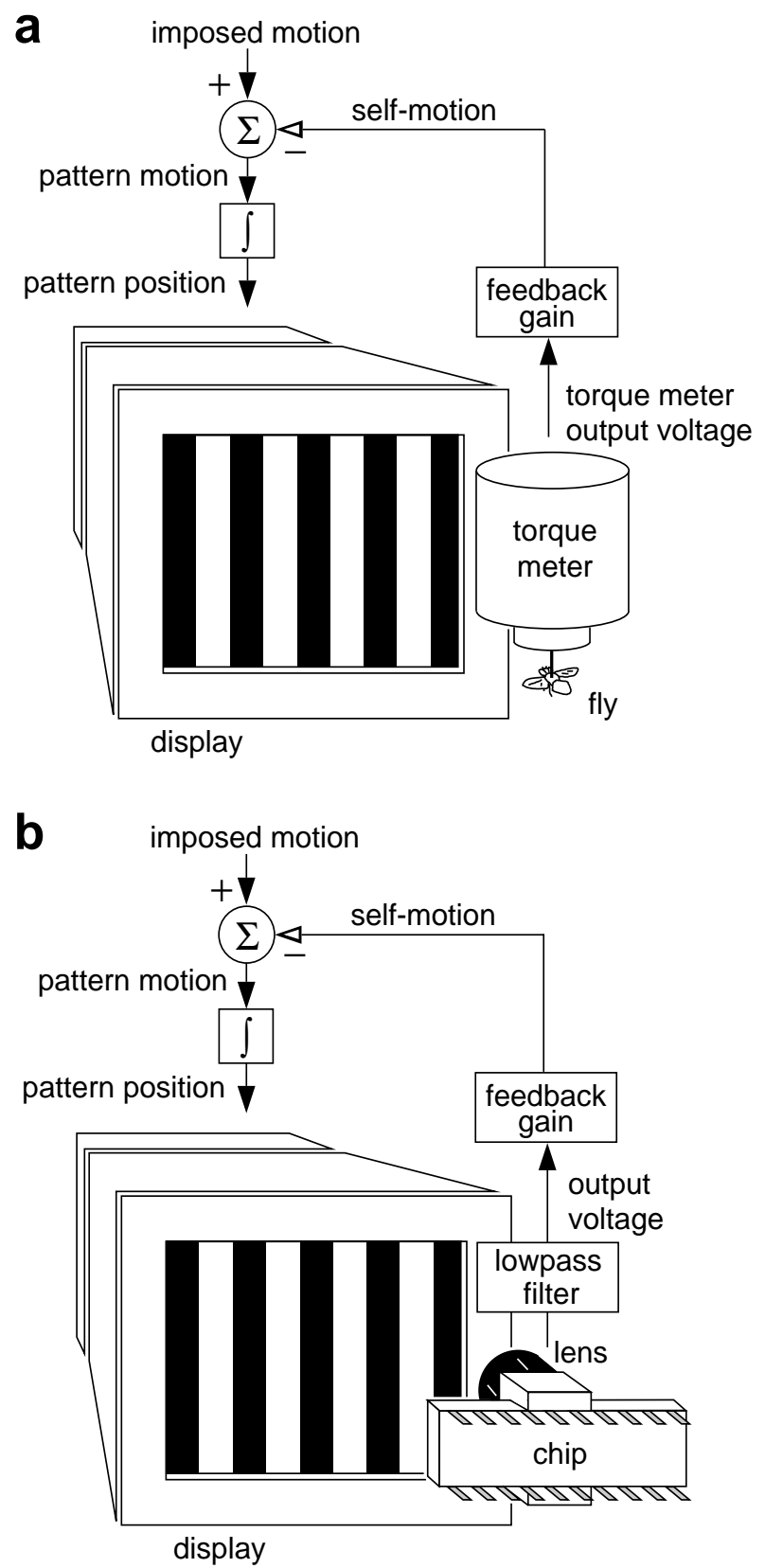
tions are not observed under open-loop conditions, suggesting they arise from optomotor feedback (Geiger \& Poggio, 1981; Warzecha \& Egelhaaf, 1996). Notice that despite the large amplitude of the torque oscillations, the position trace is not dominated by this effect. This fluctuation amplitude, in terms of number of photoreceptors, is close to the amplitude observed in human microsaccades (Warzecha \& Egelhaaf, 1996). Poggio and colleagues observed similar oscillations in closed-loop experiments and proposed that they arose from the 60-75 ms synaptic delay inherent in the fly visual system (Geiger \& Poggio, 1981; Poggio \& Reichardt, 1981).

3.2 Duplicating Experiments with the Silicon System. We were able to replicate these experiments with our silicon analog of the optomotor system (see Figure $4 b$ ). To provide visual stimulation, we used an LED display with a $200 \mathrm{~Hz}$ refresh rate, which is currently being used to test flies in closed-loop experiments. The stimulation time schedule was identical to the fly experiments, but an angular velocity of 50 degrees $\mathrm{s}^{-1}$ was used. Our chip had a much smaller field of view (10 degrees) than the fly's, so we set the stimulus distance such that the EMD array saw approximately one wavelength of the pattern. The output signal from the silicon model of the HS cell was passed through an off-chip firstorder low-pass filter with a time constant of $680 \mathrm{~ms}$, modeling the behavior of the thoracic motor centers (Egelhaaf, 1987; Wolf \& Heisenberg, 1990; Warzecha \& Egelhaaf, 1996). The filtered output of the chip was treated exactly like the signal from the torque meter in the fly experiments, and closed-loop experiments were run in real time. Figure $5 c$ shows torque and position data from the chip for an individual trial, and Figure 5d shows the averaged response over 100 trials.

The silicon system shows the same ability to cancel the imposed motion greatly. The fly showed an average drift of $9.4 \%$ of the open-loop drift velocity, with position fluctuations of 7.8 degrees (standard deviation) about this drift. The chip showed an average drift of $22 \%$ of the open-loop drift velocity, with position fluctuations of 6.2 degrees (S.D.) about this drift. Also, we observe the same $2 \mathrm{~Hz}$ oscillations in the individual trials. Since we did not build any explicit delay into our system, this demonstrates that the phase lags and nonlinearities in this simple model are sufficient to produce oscillations, even in the absence of additional synaptic delays. In future experiments, we hope to explore how these parameters determine the oscillation frequency and amplitude.

Figure 4: Facing page. Experimental methodology. (a) Setup used by Warzecha and Egelhaaf (1996) to measure the closed-loop torque response of the sheepfly Lucilia. The torque meter output is scaled to produce a measure of what the fly's self-motion would be if it were free to rotate. This self-motion is subtracted from the imposed motion to determine the pattern motion, creating the illusion of free flight in a room with distant walls. (Only rotation, not translation, is simulated.) (b) Setup used to replicate the closed-loop experiments with the silicon model. The output voltage from the circuit is used in place of the torque meter output voltage. The rest of the system is identical to $a$. 


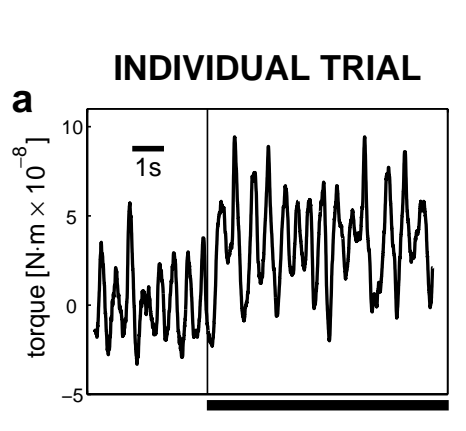

\section{FLY}
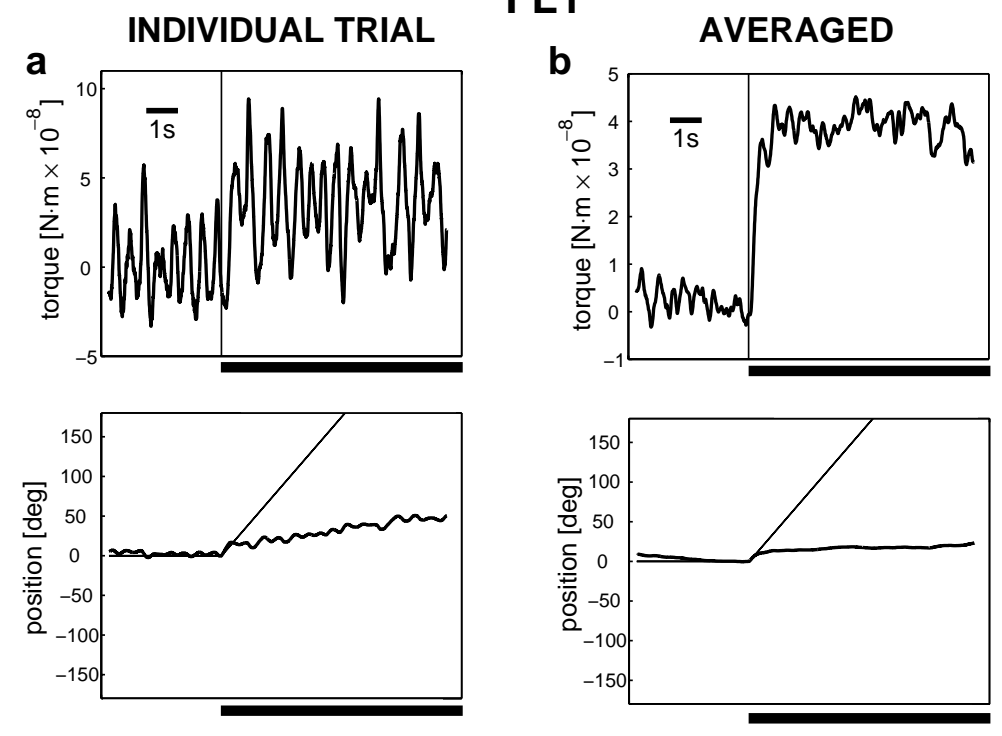

SILICON
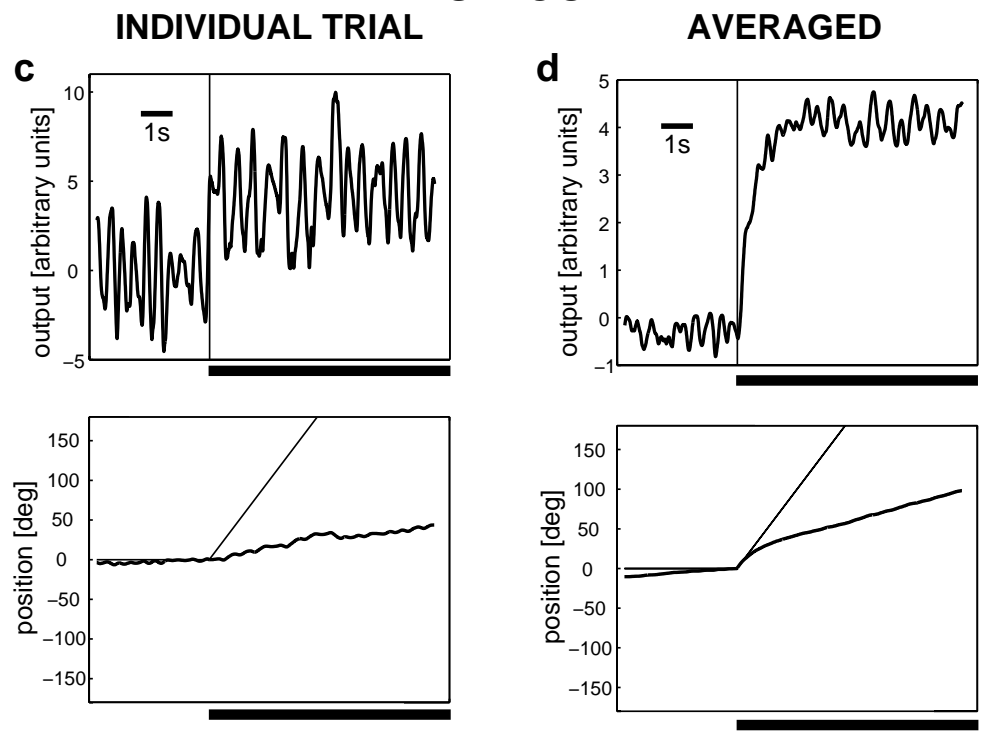


\section{Conclusion}

We have demonstrated a small, power-efficient silicon system built in a standard CMOS process that replicates behavior observed in a biological sensorimotor control system. Our hardware model senses the image and generates motor commands in real time, allowing direct, quantitative comparisons to biological systems to be made using the same experimental apparatus.

There are still important differences between our hardware model and the biological system. For example, the temporal frequency sensitivity of the silicon system is much narrower than the sensitivity of lobular plate neurons (see Figure 3). Future instantiations might use spatial instead of temporal bandpass filtering to remove the DC component from the input image while maintaining temporal sensitivity. Also, time-constant adaptation in the motion detectors could be implemented and explored. It would be interesting to observe visually guided behaviors in a hardware model with adaptation enabled or disabled. This experiment might be difficult or impossible to achieve with a real animal and might lend insight into the benefits of adaptation in sensorimotor systems.

We believe this hardware modeling approach will prove increasingly valuable in the future, as biological models of the neural circuitry underlying more complex and sophisticated behaviors arise. To simulate a sensorimotor system in software, one must construct two models: a model of the biological system and a model of the world. The physical environment is an essential element in a sensorimotor feedback loop, so this world model must increase in detail as we study more advanced behaviors. Since animals interact with their three-dimensional environment in very dynamic ways, it may not be long before software simulations of sensorimotor systems require more computational resources to model the world than to model the neural circuitry of interest.

By using a physically instantiated hardware model with integrated sensors, we can replicate experiments using existing stimuli developed for studying

Figure 5: Facing page. Comparing the fly's optomotor behavior to our silicon system. (a) Torque (top panel) and angular position (bottom panel) versus time for an individual closed-loop trial with a fly. The dark horizontal bar indicates experimenter-imposed rotation. Thin lines on the position trace indicate position in the open-loop case. Most of the imposed rotation is cancelled out by the optomotor control system. Since the position is proportional to the integral of the torque (see text for details), large torque oscillations do not cause large position oscillations. (b) Averaged torque response and angular position trace for multiple trials ( $N=139,5$ flies). The fly showed an average drift of $9.4 \%$ of the open-loop drift velocity, with position fluctuations of 7.8 degrees (standard deviation) about this drift. (c) Chip output signal (analogous to torque) and position versus time for the silicon system in an individual trial. (d) Averaged torque response and angular position trace for multiple trials $(N=100,1$ chip). The chip showed an average drift of $22 \%$ of the open-loop drift velocity, with position fluctuations of 6.2 degrees (S.D.) about this drift. Data in $a$ and $b$ redrawn from Warzecha and Egelhaaf (1996). 
animals. This approach also opens up the possibility of endowing our systems with real motor capabilities, an obvious extension of the work presented here. We could conceivably build mobile robots and test them in complex environments that would be extremely difficult to model in software. Neural models may also be implemented in software and run on digital computers using traditional CCD cameras, but if sensorimotor feedback is of interest, the software must run in real time. If mobile robotic systems are used, then the size and power advantages of the analog VLSI approach presented here would be especially beneficial.

We believe that neuromorphic engineering represents a new tool for understanding complex biological systems and as a testbed for evaluating how theoretical models perform in low-accuracy hardware embedded into a noisy world. Many of the neural systems being studied involve real-time sensory processing and motor control that greatly exceed the capabilities of modern digital computers. As we implement biological models in compact, power-efficient ways, this approach may have significant engineering uses as well.

\section{Acknowledgments}

We thank M. Egelhaaf, A.-K. Warzecha, J. Haag, and B. Kimmerle for invaluable advice and assistance and for the use of their resources; and B. Minch, H. Krapp, R. Deutschmann, and E. Slimko for useful discussions. This work was supported by the NSF Center for Neuromorphic Systems Engineering Research Center, grants from ONR, and an NDSEG fellowship (R.H.).

\section{References}

Adelson, E. H., \& Bergen, J. R. (1985). Spatiotemporal energy models for the perception of motion. J Opt Soc Am A, 2, 284-299.

Andreou, A., \& Strohbehn, K. (1990). Analog VLSI implementation of Hassenstein-Reichardt-Poggio models for vision computation. In Proc 1990 IEEE Symp Syst, Man, Cybern (pp. 707-710).

Borst, A., \& Egelhaaf, M. (1987). Temporal modulation of luminance adapts time constant of fly movement detectors. Biol Cybern, 56, 209-215.

Borst, A., \& Egelhaaf, M. (1989). Principles of visual motion detection. TINS, 12, 297-305.

Borst, A., Egelhaaf, M., \& Haag, J. (1995). Mechanisms of dendritic integration underlying gain control in fly motion-sensitive interneurons. J Comput Neurosci, 2, 5-18.

Clifford, C. W. G., Ibbotson, M. R., \& Langley, K. (1997). An adaptive Reichardt detector model of motion adaptation in insects and mammals. Visual Neuroscience, 14, 741-749.

Delbrück, T. (1993). Silicon retina with correlation-based, velocity-tuned pixels. IEEE Trans Neural Networks, 4, 529-541.

Delbrück, T., \& Mead, C. (1996). Analog VLSI phototransduction by continuoustime, adaptive, logarithmic photoreceptor circuits (CNS Memo No. 30). Pasadena: California Institute of Technology. 
de Ruyter van Steveninck, R. R., Zaagman, W. H., \& Mastebroek, H. A. K. (1986). Adaptation of transient responses of a movement-sensitive neuron in the visual system of the blowfly Calliphora erythrocephala. Biol Cybern, 54, 223236.

Egelhaaf, M. (1987). Dynamic properties of two control-systems underlying visually guided turning in house-flies. J Comp Physiol A, 161, 777-783.

Egelhaaf, M., \& Borst, A. (1992). Are there separate ON and OFF channels in fly motion vision? Visual Neuroscience, 8, 151-164.

Egelhaaf, M., \& Borst, A. (1993). A look into the cockpit of the fly: Visual orientation, algorithms, and identified neurons. J Neurosci, 13, 4563-4574.

Egelhaaf, M., Hausen, K., Reichardt, W., \& Wehrhahn, C. (1988). Visual course control in flies relies on neuronal computation of object and background motion. TINS, 11, 351-358.

Etienne-Cummings, R., \& Van der Spiegel, J. (1996). Neuromorphic vision sensors. Sensors and Actuators A, 56, 19-29.

Franceschini, N., Pichon, J. M., \& Blanes, C. (1992). From insect vision to robot vision. Phil Trans $R$ Soc Lond B, 337, 283-294.

Franceschini, N., Riehle, A., \& le Nestour, A. (1989). Directionally selective motion detection by insect neurons. In Stavenga \& Hardie (Eds.), Facets of vision. Berlin: Springer-Verlag.

Geiger, G., \& Nässel D. R. (1981). Visual orientation behaviour of flies after selective laser beam ablation of interneurones. Nature, 293, 398-399.

Geiger, G., \& Nässel, D. R. (1982). Visual processing of moving single objects and wide-field patterns in flies: Behavioral analysis after laser-surgical removal of interneurons. Biol Cybern, 44, 141-149.

Geiger, G., \& Poggio, T. (1981). Asymptotic oscillations in the tracking behaviour of the fly Musca domestica. Biol Cybern, 41, 197-201.

Harrison, R. R, \& Koch, C. (1998). An analog VLSI model of the fly elementary motion detector. In M. I. Jordan, M. J. Kearns, \& S. A. Solla (Eds), Advances in neural information processing systems, 10 (pp. 880-886). Cambridge, MA: MIT Press.

Hassenstein, B., \& Reichardt, W. (1956). Systemtheoretische Analyse der Zeit, Reihenfolgen, und Vorzeichenauswertung bei der Bewegungsperzepion des Rüsselkäfers Chlorophanus. Z Naturforsch, 11b, 513-524.

Hausen, K. (1981). Motion sensitive interneurons in the optomotor system of the fly: I. The horizontal cells: Structure and signals. Biol Cybern, 45, 143-156.

Hausen, K. (1982). Motion sensitive interneurons in the optomotor system of the fly: I. The horizontal cells: Receptive field organization and response characteristics. Biol Cybern, 46, 67-79.

Hausen, K. (1984). In The lobule-complex of the fly: Structure, function, and significance in behaviour. M. A. Ali (Ed.), Photoreception and vision in invertebrates (pp. 523-559). New York: Plenum.

Hausen, K., \& Wehrhahn, C. (1990). Neural circuits mediating visual flight control in flies: II. separation of two control-systems by microsurgical brainlesions. J Neurosci, 10, 351-360.

Hengstenberg, R. (1982). Common visual reponse properties of giant vertical cells in the lobula plate of the blowfly Calliphora. J Comp Physiol A, 149, 179- 
193.

Himmelbauer, W., Furth, P. M., Pouliquen, P. O., \& Andreou, A. G. (1996). Logdomain filters in subthreshold MOS (Tech. Rep.). Baltimore: Johns Hopkins University.

Krapp, H. G., \& Hengstenberg, R. (1996). Estimation of self-motion by optic flow processing in single visual interneurons. Nature, 384, 463-466.

Lewis, M. A. (1998). Visual navigation in a robot using zig-zag behavior. In M. I. Jordan, M. J. Kearns, \& S. A. Solla (Eds.), Advances in neural information processing systems, 10 (pp. 822-828). Cambridge, MA: MIT Press.

Mead, C. (1989). Analog VLSI and neural systems. Reading, MA: Addison-Wesley.

Minch, B. A., Diorio, C., Hasler, P., \& Mead, C. (1996). Translinear circuits using subthreshold floating-gate MOS transistors. Analog Int Circuits and Signal Processing, 9, 167-179.

Moini, A., Bouzerdoum, A., Eshraghian, K., Yakovleff, A., Nguyen, X. T., Blanksby, A., Beare, R., Abbott, D., \& Bogner, R. E. (1997). An insect visionbased motion detection chip. IEEE J Solid-State Circuits, 32, 279-284.

O'Carroll, D. C., Bidwell, N. J., Laughlin, S. B., \& Warrant, E. J. (1996). Insect motion detectors matched to visual ecology. Nature, 382, 63-66.

Poggio, T., \& Reichardt, W. (1981). Visual fixation and tracking by flies: Mathematical properties of simple control systems. Biol Cybern, 40, 101-112.

Reichardt, W., \& Egelhaaf, M. (1988). Properties of individual movement detectors as derived from behavioural experiments on the visual system of the fly. Biol Cybern, 58, 287-294

Reichardt, W., \& Poggio, T. (1976). Visual control of orientation behaviour in the fly. Quarterly Reviews of Biophysics, 9, 311-375.

Sarpeshkar, R., Bair, W., \& Koch, C. (1993). Visual motion computation in ana$\log$ VLSI using pulses. In S. J. Hanson, J. D. Cowan, \& C. L. Giles (Eds.), Advances in neural information processing systems, 5 (pp. 781-788). San Mateo, CA: Morgan Kaufmann.

Single, S., Haag, J., \& Borst, A. (1997). Dendritic computation of direction selectivity and gain control in visual interneurons. J Neurosci, 17, 6023-6030.

Srinivasan, M. V., Chahl, J. S., \& Zhang, S. W. (1997). Robot navigation by visual dead-reckoning: inspiration from insects. Intl J Patt Recog and Art Int, 11, 35-47.

Warzecha, A-K., \& Egelhaaf, M. (1996). Intrinsic properties of biological motion detectors prevent the optomotor control system from getting unstable. Phil Trans R Soc Lond B, 351, 1579-1591.

Weckström, M., Juusola, M., \& Laughlin, S. B. (1992). Presynaptic enhancement of signal transients in photoreceptor terminals in the compound eye. Proc $R$ Soc Lond B, 250, 83-89.

Wolf, R., \& Heisenberg, M. (1990). Visual control of straight flight in Drosophila melanogaster. J Comp Physiol A, 167, 269-283.

Received December 17, 1998; accepted January 26, 2000. 